\title{
MÉTODOS DE COLETA DE SÊMEN EM FELÍDEOS
}

\author{
Rodrigo Neca Ribeiro ${ }^{1}$, Anna Raquel Grimas Almeida ${ }^{1}$, Antonio Campanha \\ Martinez ${ }^{2}$ \\ 1 Mestrando do Programa de Pós Graduação em Produção Sustentável e Saúde \\ Animal - UEM - Umuarama, rodrigonribeiro@hotmail.com \\ 2 Docente do Programa de Pós-Graduação em Produção Sustentável e Saúde \\ Animal - UEM - Umuarama
} Recebido em: 06/04/2019 - Aprovado em: 10/06/2019 - Publicado em: 30/06/2019
DOl: 10.18677/EnciBio_2019A88

\begin{abstract}
RESUMO
Com a destruição dos biomas em decorrência das atividades humanas houve uma exposição a riscos a biodiversidade do planeta. Desta forma nos últimos anos houve um aumento nos estudos com gatos domésticos relacionados a criopreservação de sêmen. O método de coleta de sêmen de felídeos é desafiador, devido ao pequeno volume de ejaculado e baixa concentração de espermatozoides vistos em espécies exóticas ou gatis. Existem três métodos que são normalmente realizados. A eletroejaculação é um método comumente escolhido para coleta de sêmen em felídeos, no entanto para sua realização é necessário o uso de equipamentos específicos. O outro método é a coleta de sêmen por meio de vagina artificial, mas não é uma maneira prática. E a coleta farmacológica com cateterização uretral. Tais ferramentas possibilitam a criopreservação de sêmen e o armazenamento a longo prazo de materiais biológicos de cunho genético, que irão permitir a conservação de espécies e manter a variabilidade genética. $O$ objetivo dessa revisão de literatura é citar os métodos de coletas e criopreservação de sêmen dos felídeos.
\end{abstract}

PALAVRAS-CHAVE: criopreservação, felídeos, sêmen.

\section{METHODS OF SEMEN COLLECTION IN FELID}

\section{ABSTRACT}

With the emission of biomes, it emerged from the applications of an exposition on risks to the planet's biodiversity. Thus, in recent years there has been a growth in studies with cats and animals related to cryopreservation of semen. The method of collecting semen from spermatozoa is responsible for the volume of ejaculate and low concentration of spermatozoa seen in exotic species or gatis. Like the methods that are normally executed. Electroejaculation is a commonly chosen method for collecting specimens in felids, however, it requires the use of specific equipment. Another method is the collection of semen by artificial vagina, but it is a practical way. And pharmacological research with urethral catheterization. Such tools have enabled the cryopreservation of semen and the storage of biological material data, which allows the conservation of species and maintain a genetic variability. The evaluation of a review of cite methods of collections and cryopreservation of feline semen. 
KEYWORDS: felids, semen, cryopreservation.

\section{INTRODUÇÃO}

Nos últimos anos houve um aumento nos estudos com gatos domésticos relacionados a criopreservação de sêmen. Estas pesquisas são desenvolvidas visando à compreensão e preservação de felídeos silvestres em extinção. $O$ conhecimento da função reprodutiva dos gatos domésticos é de grande importância devido a semelhança entre a anatomia reprodutiva do Felis catus e da maioria dos felinos selvagens. (DIAGONE, 2009; SILVA et al., 2009; JOHNSON, 2018).

Com a destruição dos biomas em decorrência das atividades humanas houve uma exposição a riscos a biodiversidade do planeta. Pois as populações de diferentes espécies animais diminuíram, devido a fragmentação de habitat, resultado da construção de barreiras físicas como estradas e produção agrícolas, o que afetou os limites de ocupação dos animais e sua habilidade em achar parceiros para reprodução, o que pode levar ao aumento da heterose e perdas da diversidade genética nas populações (POPE, 2000).

O método de coleta de sêmen de felídeos é desafiador, devido ao pequeno volume de ejaculado e baixa concentração de espermatozoides vistos em espécies exóticas ou gatis. Tal ferramenta possibilita o armazenamento a longo prazo de materiais biológicos de cunho genético, que irão permitir a conservação de espécies e manter a variabilidade genética (MARTINS; JUSTINO, 2015), tirando os animais da zona de risco. Existe um benefício intelectual e prático em ter um banco de reserva genética local, diminuindo a necessidade de transporte internacional e o risco de introdução de doenças exóticas (SILVA et al., 2012).

A eletroejaculação é um procedimento habitualmente escolhido para coleta de sêmen em felídeos, no entanto para sua realização é necessário o uso de equipamentos específicos e anestesia geral. A coleta de sêmen por vagina artificial, também é bastante aplicada, mas somente pode ser realizada com animais previamente treinados, o que em felídeos selvagens se torna impossível. E coleta com contenção farmacológica seguida de cateterização uretral.

As metodologias de reprodução assistida, como a criopreservação de gametas, a inseminação artificial e a fertilização in vitro, permitem a passagem apenas do material genético entre as populações de animais, podendo ser utilizada para animais domésticos e selvagens, de vida livre ou entre animais de vida livre e cativeiro (ARAUJO, 2016).

Antes da realização da coleta de sêmen, deve-se realizar um exame físico geral, com o objetivo de avaliar as condições gerais do animal. Podendo incluir até exames complementares como hemograma completo e perfil bioquímico. Em seguida deve ser realizado um exame específico do aparelho reprodutivo que inclui palpação manual dos testículos, devem ser medidos em seu comprimento, largura e altura, se é móvel e sua consistência. O pênis de ser avaliado quanto a cor, ejaculado e a presença de espículas. É mais fácil a exteriorização do pênis após a sedação (JOHNSON, 2018). O objetivo dessa revisão de literatura é citar os métodos eficazes de coletas para a criopreservação de sêmen dos felídeos.

\section{MÉTODOS PARA REALIZAÇÃO DE COLETA DE SÊMEN Eletroejaculação}

A eletroejaculação é a técnica comumente escolhida para coleta de sêmen em felídeos, no entanto para sua realização é necessário o uso de equipamentos 
específicos, operador treinado, do contrário pode resultar em amostras de sêmen diluídas e também contaminadas com urina (ZAMBELLI et al., 2008; LUEDERS et al., 2012). Outro fato é o uso de anestésicos adequados.

O primeiro uso de eletroejaculação em felídeos foi em gatos domésticos na década de 70 (SILVA et al., 2011). Protocolos de eletroejaculação já foram desenvolvidos para pelo menos 28 espécies de felídeos (SILVA et al., 2011). O protocolo de ejaculação descrito por Howard (1993) é amplamente utilizado em inúmeras espécies de felídeos selvagens como tigre, onça parda, jaguatirica e onça pintada (DECO-SOUZA et al., 2010; ÁVILA et al., 2012; ERDMANN, 2013).

Para realizar o método de eletroejaculação é necessário o uso de contenção química, com a finalidade de evitar riscos a equipe e ao animal (Figura 1). A qualidade da anestesia deve ser suficiente para executar a colheita de sêmen e promover analgesia ao animal do início ao fim do procedimento (ERDMANN, 2005).

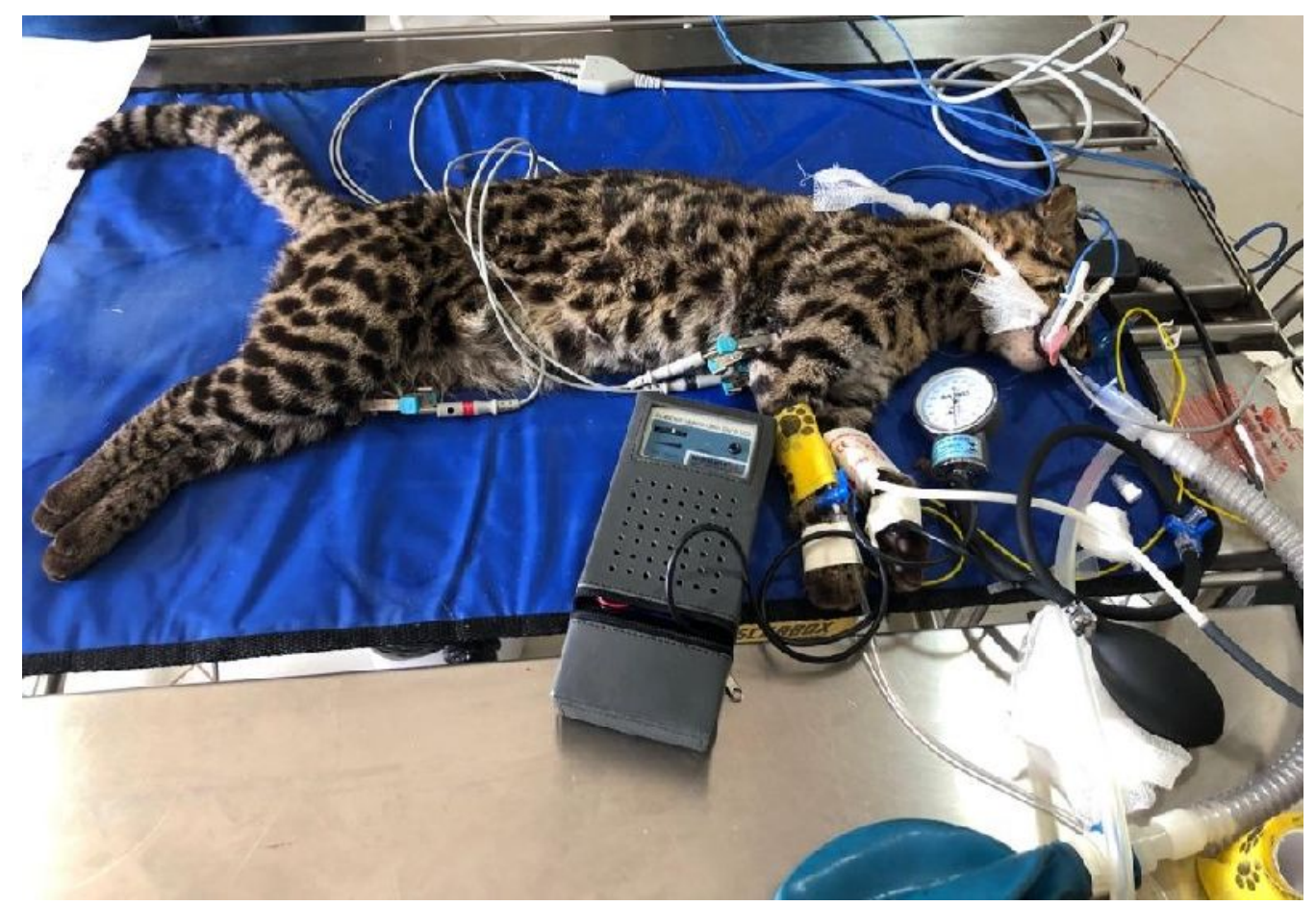

FIGURA - 1 Sedação do animal para realização de coleta seminal (Arquivo Pessoal).

A anestesia sempre apresenta risco em qualquer idade do animal. Antes da administração dos fármacos anestésicos, alguns fatores devem ser considerados, incluindo o estado de saúde, a atitude e peso corporal. Além das categorias de risco anestésico e estado físico avaliados de acordo com a Sociedade Americana de Anestesiologistas que de termina a seguinte classificação: ASA I Paciente normal e hígido; ASA II Paciente com doença sistêmica de grau leve; ASA III Paciente com doença sistêmica grave; ASA IV Paciente com doença sistêmica grave que é uma ameaça constante à vida; ASA V Paciente moribundo, sem expectativa de sobrevivência sem cirurgia e por fim o ASA VI Paciente com morte cerebral cujos 
órgãos serão removidos para fins de doação (ASA, 2014). De forma ideal, devem ser realizado exames pré-anestésicos como hemograma completo, perfil renal e hepático.

Os estímulos elétricos do protocolo podem gerar desconforto e sensibilidade muscular aos animais. Também promove relaxamento muscular vesical o que poderá causar a contaminação do sêmen por urina (ERDMANN, 2013). Voltagens mais elevadas e posicionamento muito cranial do transdutor retal aumentam a chance de contaminação por urina no procedimento (MOREIRA, 2017).

Hideo Tajima et al., (2016) coletaram sêmen de leopardo usando o método de eletroejaculação transretal e investigaram as qualidades do sêmen por cerca de quatro anos. A influência da estação na função espermatogênica destes animais foi investigada em relação a qualidade do sêmen, volume testicular e nível sérico de testosterona. Como resultado, puderam coletar sêmen com boas qualidades espermática que seriam úteis para a inseminação artificial. Alguma sazonalidade foi observada no volume testicular e no nível sérico de testosterona. Notaram que as características eram favoráveis antes e durante a época de reprodução em comparação com as que ocorreram após a época reprodutiva.

Desse modo, o aperfeiçoamento de técnicas mais práticas de coleta de sêmen é de grande valia no desenvolvimento de programas de reprodução (ARAUJO, 2016). Pires et. al (2010) avaliaram a anestesia epidural com lidocaína após a indução com propofol, bem como seus efeitos cardiorrespiratórios, como uma alternativa à anestesia dissociativa para eletroejaculação em gatos domésticos. $\mathrm{O}$ protocolo proposto mostrou-se eficiente por proporcionar sedação e analgesia adequadas, por não induzir ao estresse, e não interferir na qualidade seminal e por permitir, devido ao seu metabolismo, uma recuperação rápida e tranquila dos gatos.

Existem vários modelos de aparelhos, alguns utilizam corrente elétrica contínua, outras alternada. O aparelho pode ser fabricado sob medida para uso em felinos ou pode-se adquirir o mesmo tipo utilizado para bovinos.

A sonda deve possuir o tamanho adequado ao porte da espécie, sendo aproximadamente do diâmetro das fezes do animal. Para carnívoros, a que permitiu melhores respostas ejaculatórias foram as que possuíam tiras longitudinais ao invés de circulares (ERDMANN, 2014). Fukui et.al (2013) relataram os efeitos da eletroejaculação durante 6 anos em tigres siberianos, mostrando que a motilidade tende a aumentar com o número de coletas frequentes.

Um experimento realizado com leopardo árabe, cujo o objetivo era avaliar a morfologia espermática e a qualidade da ejaculação em machos cativos, foi realizado com oito animais (de 2 a 16 anos) durante os meses de verão e inverno. Os leopardos de dois anos de idade apresentaram menor concentração de espermatozóides por ejaculado, ao serem comparados com os animais mais velhos. Os ejaculados coletados durante 0 verão, mostraram concentrações significativamente mais baixas de espermatozoides, contagem total de espermatozóides, motilidade espermática e viabilidade e porcentagem de espermatozóides mostrando morfologia normal do que os ejaculados coletados no inverno mais frio. Os resultados mostraram que o leopardo atinge a maturidade sexual entre 2 e 3 anos de idade e exibe boa qualidade de sêmen até 8 anos (DORSSER; STRICK, 2005). 


\section{Procedimento para a eletroejaculação}

- $\quad$ O método de coleta por eletroejaculação é realizado por sonda retal de $1 \mathrm{~cm}$ de diâmetro e 12 a $13 \mathrm{~cm}$ de comprimento. O modelo está representado na Figura 2.

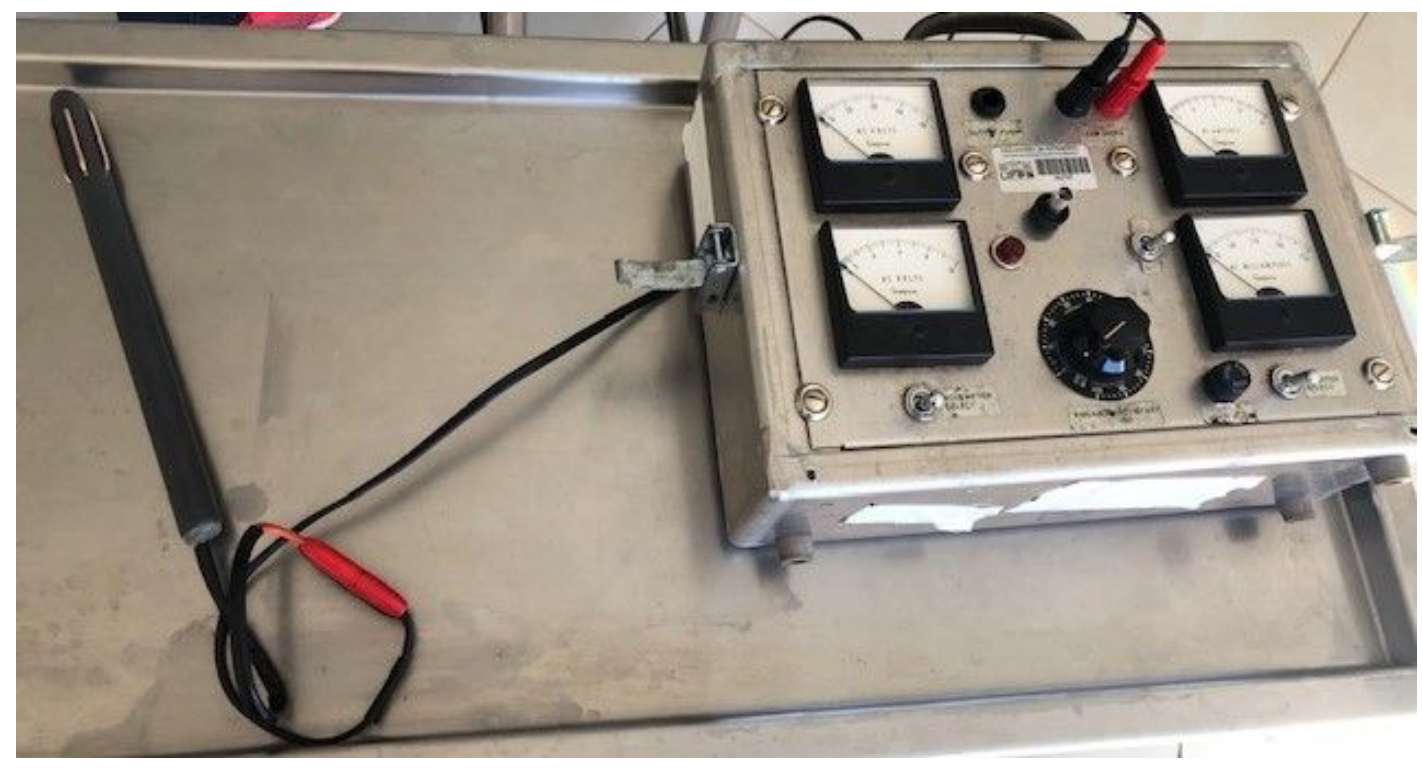

FIGURA 2 - Sonda retal de eletroejaculação felina, medida $1 \mathrm{~cm}$ e 12 a $13 \mathrm{~cm}$ de comprimento e aparelho que emite os estímulos (Arquivo pessoal).

- Verificar se há fezes na ampola retal, caso tenha, com o dedo enluvado e lubrificado deve-se evacuar o reto.

- A sonda retal deve ser lubrificada, em seguida a sonda deve ser inserida levemente no reto do animal, por aproximadamente $5 \mathrm{a} 7 \mathrm{~cm}$. Os eletrodos devem ser posicionados ventralmente (Figura 3). 


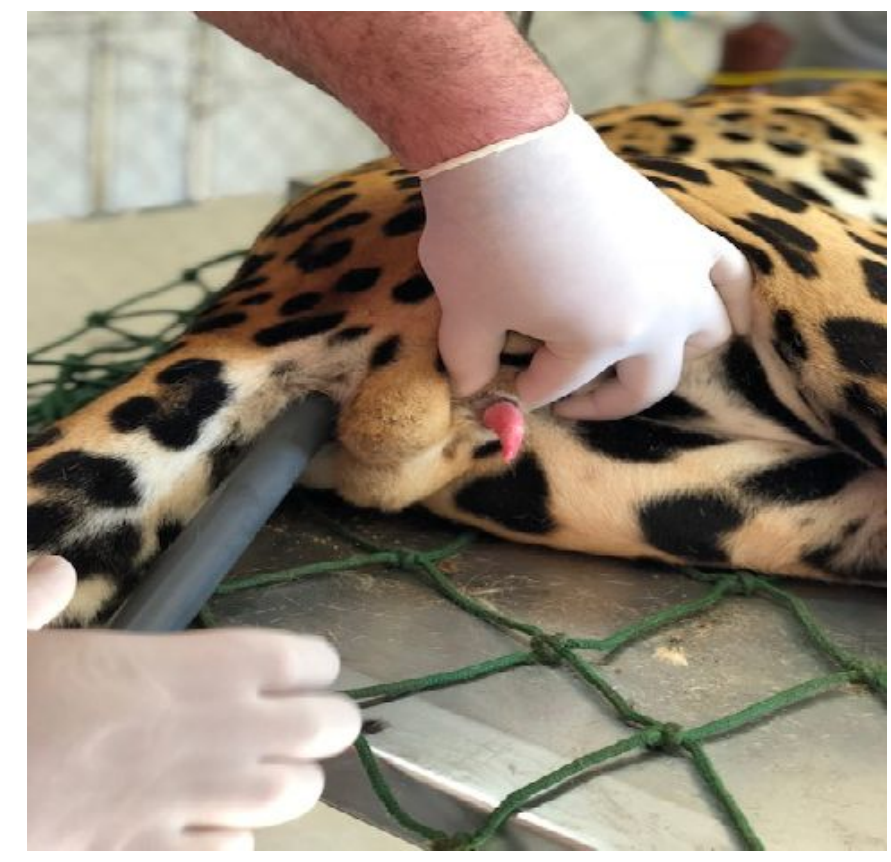

FIGURA - 3 Introdução da sonda retal para realização da eletroejaculação (Arquivo pessoal).

- $\quad$ Com o auxílio de uma gaze, o pênis deve ser exposto manualmente e limpo com solução fisiológica, depois deve ser secado.

- $\quad$ Posicione um frasco estéril (Eppendorf) sobre o pênis e o direcione ventralmente. Isso permite que o ejaculado entre no tubo, evitando contaminação (figura 3).

- Antes de ligar o eletroejaculador, certifique-se de que o disco do reostato esteja desligado e ajustado em zero.

- $\quad$ Gire o reostato para fornecer uma série de estímulos elétricos girando gradualmente o mostrador para a voltagem desejada por 2 a 3 segundos, e então retorne para o zero por 2 a 3 segundos. Os estímulos dever ser administrado da seguinte forma:

Ajuste 1: 10 vezes a $2 \mathrm{~V}, 10$ vezes a $3 \mathrm{~V}, 10$ vezes a $4 \mathrm{~V}$, o animal deve descansar por 3 a 5 minutos;

Ajuste 2: 10 vezes a $3 \mathrm{~V}, 10$ vezes a $4 \mathrm{~V}, 10$ vezes a $5 \mathrm{~V}$, o animal deve descansar de 3 a 5 minutos;

Ajuste $3: 10$ vezes a $4 \mathrm{~V}, 10$ vezes a $5 \mathrm{~V}, 10$ vezes a $5 \mathrm{~V}$ (ou 6 , se necessário, dependendo da resposta anterior. 


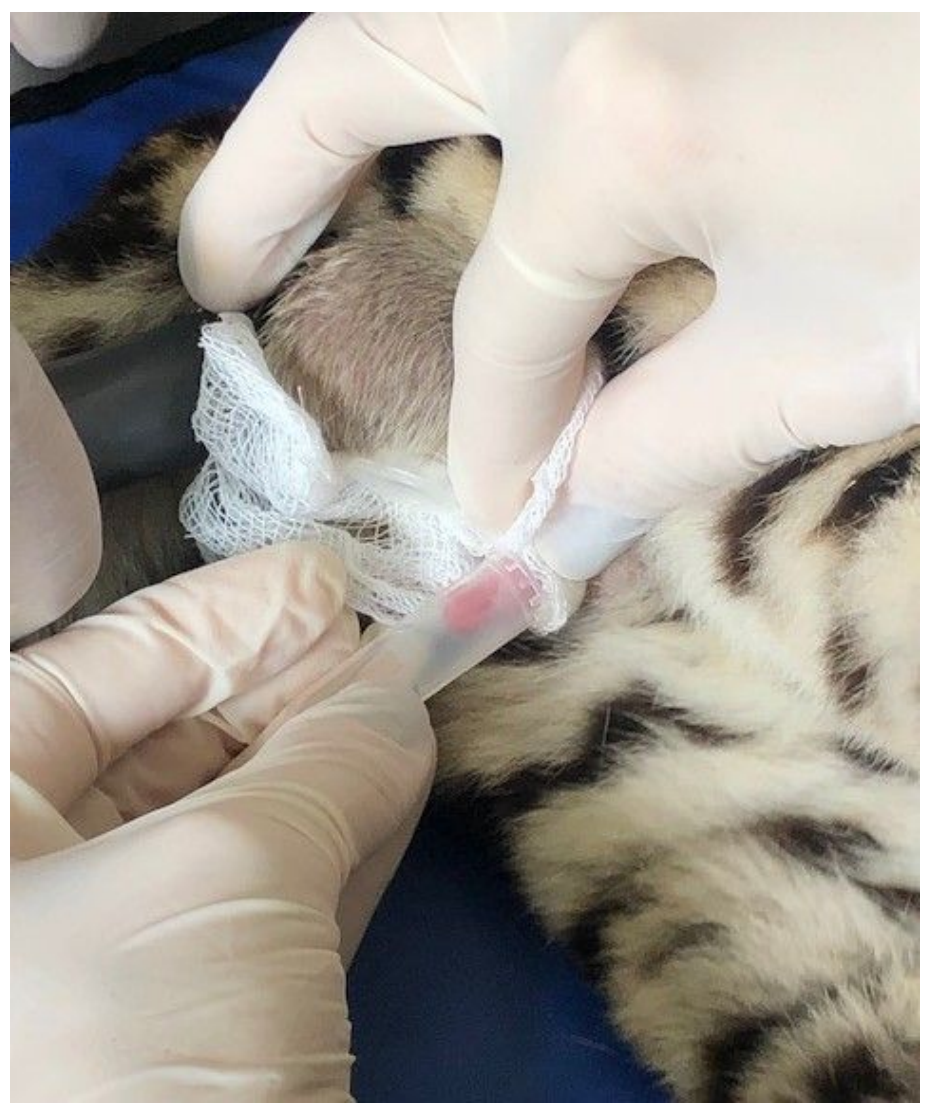

FIGURA 4 - Para o método de eletroejaculação, um Eppendorf estéril é colocado sobre o pênis ereto e posicionado ventralmente. A alça da sona retal também pode ser vista sendo inserida no reto (Arquivo pessoal).

Após a coleta o ejaculado deve ser avaliado de acordo com as características físicas (volume, aspecto, turbilhão, motilidade, vigor e concentração) e morfológicas (defeitos maiores, menores e totais) dos ejaculados segundo o Colégio Brasileiro de Reprodução Animal.

\section{Vagina artificial}

A coleta de sêmen por vagina artificial tem sido empregada por ser uma técnica de baixo custo. No entanto não é um método prático de avaliação andrológica de animais selvagens, pois existe a necessidade do treinamento de duas a três semanas do animal e este treinamento é bem-sucedido em somente 60 a $70 \%$ dos casos. $O$ gato doméstico mesmo que treinado, se colocado em ambiente que não é o seu usual, provavelmente não ejaculará (AXNÉR; LINDE-FORSBERG, 2002). Entretanto, alguns gatos com temperamento mais agressivo e em felídeos selvagens, esta é uma prática que oferece inúmeros riscos às pessoas que irão manipular o animal (STORNELLI, 2001).

A coleta de sêmen com vagina artificial permite um ejaculado perfeito para análise. $O$ equipamento pode ser montado com um tubo de Eppendorf e um bulbo de borracha. É permitido que o macho monte uma fêmea em cio, a vagina artificial deve ser acoplada no pênis e apoiada pelo dedo polegar, enquanto o dedo indicador estimula a ejaculação. A coleta por vagina artificial apresenta a vantagem de permitir a sua realização de forma diária, ou em intervalos curtos regulares, diferentemente 
da eletroejaculação. Intervalos de coleta dupla (2 coletas consecutivas com intervalos de 5 a 10 minutos) por vagina artificial de 24,48 e $72 \mathrm{~h}$ foram testadas quanto ao seu efeito na qualidade seminal, e demonstrou-se que a qualidade seminal não é afetada por até 10 dias de coletas diárias (TANAKA et al., 2000).

Valiente et. al. (2013) avaliaram o treinamento de gatos domésticos na ejaculação em uma vagina artificial. Cinco gatos pós-púberes foram treinados para ejacular em uma vagina artificial três vezes por semana durante 20 minutos. Cinquenta ejaculados obtidos por vagina artificial foram avaliados mostrando ser uma técnica funcional, mas que exige tempo e treinamento do animal.

\section{Coleta farmacológica de sêmen}

Recentemente, a medetomidina, um sedativo alfa-2 agonista, vem sendo usado de forma promissora na coleta de sêmen de felinos. Esta metodologia já foi testada em gatos e leões africanos, possibilitando a obtenção de amostras concentradas de sêmen em animais anestesiados (Figura 5), dispensando o eletroejaculador, sem perda da qualidade espermática. É uma técnica promissora a ser aplicada nas demais espécies de felinos (ZAMBELLI et al., 2008; LUEDERS et al., 2012).

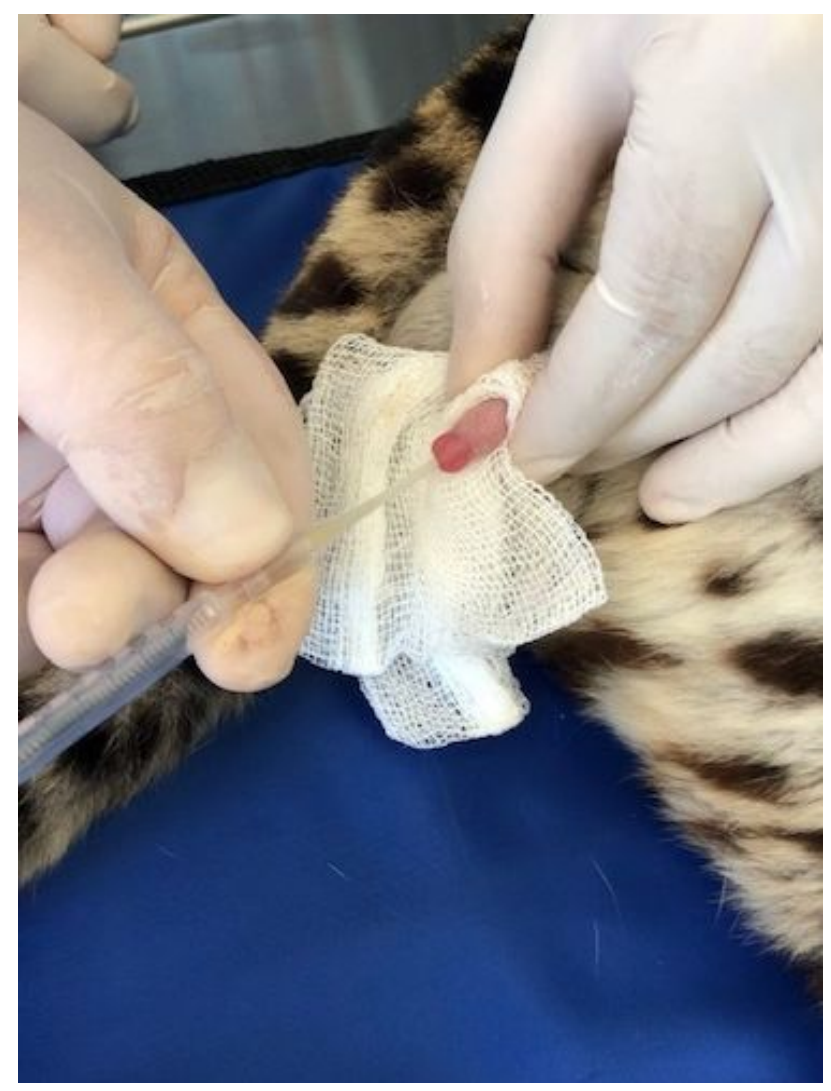

FIGURA - 5. Cateterização para coleta seminal em Leopardus wiedii (Arquivo pessoa).

Cunto et al., (2015) avaliaram a influência de diferentes protocolos de cateterização uretral após a indução farmacológica sobre a qualidade do sêmen do gato doméstico. $\mathrm{O}$ estudo foi dividido em duas etapas, uma em que diferentes doses de medetomidina administrada eram avaliadas e a etapa dois realizava-se um teste 
no momento da cateterização após a indução farmacológica. No primeiro experimento, 18 gatos foram sedados com a dose recomendada de medetomidina (130 g / kg i.m.) enquanto os outros 18 foram sedados com uma dose menor do mesmo medicamento (50 g/ kg i.m.).

No segundo experimento, os animais foram separados em três grupos, cada um contendo 25 indivíduos. No grupo 1, a coleta de sêmen foi realizada imediatamente após o efeito farmacológico da droga; no grupo 2, a coleta de sêmen foi realizada três vezes a cada 5 minutos após o efeito farmacológico; finalmente, no grupo 3, foi realizada 20 min após o efeito farmacológico ter sido alcançado.

Todos os diferentes protocolos permitiram a coleta de espermatozoides. $O$ primeiro experimento apresentou melhor qualidade em termos de volume, concentração, número total de espermatozoides e qualidade movimento em linha reta. No segundo experimento, a motilidade do primeiro grupo foi maior; o volume total foi maior no segundo e terceiro grupos, enquanto outros parâmetros não foram estatisticamente diferentes. Os resultados sugerem que um único cateterismo imediatamente após o início do efeito farmacológico leva a um sêmen de boa qualidade com a menor possibilidade de danificar a uretra e que uma sedação com $130 \mathrm{~g} / \mathrm{kg}$ de medetomidina leva a uma coleta de espermatozóides de melhor qualidade que $50 \mathrm{~g} / \mathrm{kg}$.

Kheirkhah et al., (2107), avaliaram a colheita de sêmen do gato-da-selva (Felis chaus) por cateterização uretral (CT) após administração de medetomidina, que oferece abordagens viáveis e diferentes para a obtenção de espermatozoides de boa qualidade, especialmente em felídeos silvestres.

A pesquisa realizou o seguinte método, um cateter urinário comercial para gatos (dependendo do tamanho do animal), foi introduzido na uretra para alcançar a região primária completamente dilatada do sêmen da uretra, de modo a permitir a coleta de sêmen no lúmen do cateter por forças capilares. Após a retração, os volumes espermáticos entre $69 \pm 27,92 \mathrm{~L}$ geraram motilidade de 77,13 \pm 14,15 (média \pm DP) com uma concentração média de espermatozóides de 75,13 \pm 17,05 milhões / ml. Os resultados deste estudo mostraram que a coleta de sêmen no gato da selva é viável, usando este método. Este estudo descreve um método simples, útil em campo, de baixo custo, que não requer o treinamento do animal sendo assim melhor do que outros métodos.

Lueders et al. (2012) testaram a viabilidade de uma abordagem diferente usando cateterismo uretral (UC) em sete leões africanos. Após anestesia geral com a medetomidina e cetamina, utilizaram um ultrassom transretal para localizar a próstata. Um cateter urinário de cão comercial $(2,6$ ou 3,3 $\mathrm{mm}$ de diâmetro) avançou aproximadamente $30 \mathrm{~cm}$ na uretra para permitir a coleta de sêmen no lúmen do cateter por forças capilares. Após a retração, foi colhido sêmen apresentaram uma motilidade de mais ou menos $80 \%$, com com boa concentração espermática.

Gediendson et al., (2017) avaliaram a metodologia de coleta farmacológica de sêmen com sondagem uretral em onças pardas mantidas em cativeiro. Para a coleta farmacológica foi utilizada medetomidina para induzir a liberação de sêmen na uretra e sonda uretral para gatos, sem janela lateral, para coleta do sêmen por capilaridade. O método foi eficaz em todos os animais utilizados, comprovando que esta técnica foi eficaz. Amostras com menor volume (106,7 L) e maior concentração foram coletadas. As avaliações de vigor, motilidade e morfologia espermática demonstraram que a técnica não afeta a qualidade do sêmen. Desta forma, a metodologia proposta consiste em uma técnica mais prática e eficiente na 
coleta de sêmen com boa qualidade dispensando o eletro ejaculador nesta espécie de felino.

Zambelli et al. (2007) relataram ainda que a Medetomidina em gatos não causou ereção e nem a ejaculação, mas promoveu a liberação dos espermatozoides na uretra. Esta liberação possivelmente está relacionada à estimulação dos receptores alfa-2- adrenérgicos que promovem a contração dos ductos deferentes, do trígono vesical e do esfíncter da bexiga durante a ejaculação. Desta forma, apesar da medetomidina não promover ereção ou ejaculação em felinos, ao sondar esses animais foi possível coletar o sêmen liberado na uretra. $\mathrm{O}$ uso de antagonistas específicos dos receptores adrenérgicos do tipo $\alpha-2$, possibilita o controle dos efeitos dos agonistas, sendo estes produzidos ou revertidos à semelhança dos relaxantes musculares, o que se faz de grande interesse para a anestesiologia (KAMIBAYASHI; MAZE, 2000).

A associação de de cetamina e de medetomidina para contenção quimica de leopardo também já foi relatada com êxito por TAJIMA (2016). O cateterismo uretral após a indução farmacológica com medetomidina mostrou-se útil para a coleta de sêmen em gatos domésticos. Entretanto, a maioria dos protocolos utilizados anteriormente requer a administração de altas doses de medetomidina, que podem aumentar o risco de efeitos colaterais, especialmente no sistema cardiovascular.

Alguns fatores favorecem a utilização de ejaculação farmacológica, entre eles está o uso de reversores químicos aos anestésicos utilizados. O atipamezole, o mais recente antagonista, por promover reversão dos efeitos sedativos possui meia vida de eliminação de 2 horas, coincidindo com a dexmedetomidina, sendo improvável a recorrência dos efeitos uma vez antagonizados (MARTIN-FLORES et al., 2017).

\section{Outros métodos de coleta de sêmen}

Outra alternativa para a coleta espermática e criopreservação, é por meio de lavagem ou aspiração do epidídimo, que pode ser aplicada em animais que foram submetidos à orquiectomia ou que morreram (TEBET et al, 2006; SIEMIENIUCH ;

WOCLAWEK-POTOCKA, 2008; TOYONAGA et al., 2010). Um estudo realizado por

Jiménez et al., (2011), relataram que os procedimentos anestésicos podem afetar a motilidade dos espermatozoides colhidos do epidídimo fresco, além dos outros parâmetros como a membrana, integridade do acrossomo, viabilidade espermática e integridade da membrana funcional, quando congelados e descongelados também sofreram alterações.

Ainda outro método de coleta que pode ser realizado é a aspiração ou lavagem da cúpula vaginal da gata fêmea após o acasalamento. Este método normalmente é usado apenas para determinar se há ou não presença de espermatozoide (JOHNSON, 2018).

\section{CONSIDERAÇÕES FINAIS}

Com esta revisão de literatura foi possível levantar as formas de colheita de sêmen em felídeos, avaliando suas particularidades. A coleta através da vagina artificial embora seja possível possui algumas limitações. Durante muitos anos a eletroejaculação era o método mais empregado em felídeos selvagens. Com a 
utilização de fármacos alfa 2 adrenérgicos mais seguros a coleta farmacológica de sêmen através da sondagem uretral pode ser uma ferramenta viável, prática e segura. Além de novas técnicas como a coleta por aspiração do epidídimo e a lavagem da cúpula vaginal da gata após o coito. Desta forma é importante escolher o melhor método para as diferentes espécies e assim seja possível realizar uma coleta eficaz para a criopreservação e preservação das espécies nos bancos genéticos.

\section{REFERÊNCIAS}

ARAUJO, G. R; PAULA, T. A. R.; SOUZA, T. D.; MORATO R. G.; FERREIRA, L. B. C.; SILVA L.C.; COSTA, D. S.; BRAUD, C. Comparison of semen samples collected from wild and captive jaguars (Panthera onca) by urethral catheterization after pharmacological induction. Animal Reproduction Science Received, v. 195, p. 17, 2017. Disponível em: https://doi.org/10.1016/j.anireprosci.2017.12.019

ASA physical status classification system. [s.I.]: ASA, 2014. Disponível em: https://www.asahq.org/standards-and-guidelines/asa-physical-status-classificationsystem. Acessado em: 23 Mai. 2018.

ÁVILA, E. C.; PAULA, T. A. R.; DECO-SOUZA, T.; TRINDADE, T. F. S. L.; MASCARENHAS, R. M.; et al., Protocolos de coleta de sêmen por eletroejaculação em jaguatiricas (Leopardus pardalis). Revista Brasileira de Reprodução Animal, Belo Horizonte, v. 36, n. 4, p. 260-263, 2012. Disponível em: https://cbra.websiteseguro.com/pages/publicacoes/rbra/v36n4/p260263\%20(RB418).pdf

CUNTO, M., KÜSTER, D. G., BINI, C., CARTOLANO, C., PIETRA, M., \& ZAMBELLI, D. Influence of Different Protocols of Urethral Catheterization after Pharmacological Induction (Ur.Ca.P.I.) on Semen Quality in the Domestic Cat. Reproduction in domestic animals, v. 50, n. 6, p. 999-1002, 2015. Disponivel em: https://doi.org/10.1111/rda.12626

DA PAZ, R. C. R.; MORATO, R. G.; CARCIOFI, A.; GUIMARÃES, M. A. B. V.; PESSUTTI, C.; SANTOS, E. F.; BARNABE, R. C. Nutritional influence on quality of semen of jaguar in captivity. International Zoo Yearbook, 2006.

DA PAZ R. C. R.; ZUGE R. M; BARNABE, R. C.; BARNABE, V. H. Frozen jaguar (Panthera onca) sperm capacitation and ability to penetrate zona free hamster oocytes. Brazilian Journal of Veterinary Research and Animal Science (Impresso), v. 5, p. 337-344, 2007. Disponível em: http://agris.fao.org/agrissearch/search.do?recordlD=BR2013901104

DECO, S. T.; PAULA, T. A. R.; COSTA, D. S.; ARAÚJO, G. R.; GARAY, R. M; VASCONCELOS, G. S. C.; CSERMAK JR, A. C.; SILVA, L. C.; BARROS, J. B. G. Coleta e avaliação de sêmen de pumas (Puma concolor Linnaeus, 1771) adultos mantidos em cativeiro. Revista Brasileira de Reprodução Animal, Belo Horizonte, v. $34, \quad$ n. 4 , p. 252- 259, 2010. Disponível em: http://www.cbra.org.br/pages/publicacoes/rbra/v34n4/p252-259.pdf 
DORSSER, F. J.H.V. \& STRICK J. A. Semen characteristics and sperm morphology in the Arabian leopard (Panthera pardus nimr) and how these vary with age and season Reproduction. Fertility and Development, v. 17, n. 7, p 675-682, 2005. Disponível em: https://www.ncbi.nlm.nih.gov/pubmed/16364220

ERDMANN, R. H.; MOREIRA, N.; CUBAS, Z. S.; MORAIS, W.; OLIVEIRA, M. J.; et al.,; Contenção farmacológica da jaguatirica, Leopardus pardalis, para coleta de sêmen, pela associação de tiletamina-zolazepam e xilazina. Bioscience Journal, Uberlândia, v. 29, n. 1, p. 1731-1737, 2013. Disponível em: http://www.seer.ufu.br/index.php/biosciencejournal/article/view/18003

FLORES, M. M.; D. SAKAI, J. HONKAVAARA, L. CAMPOY, D. PORTELA, R. GLEED. Cardiovascular effects of low-dose intravenous atipamezole and MK-467 in anesthetized cats receiving dexmedetomidine. Veterinary Anesthesia and analgesia, v. 44, n. 5, p. 1262, 2017. https://doi.org/10.1016/j.vaa.2017.09.023

HOWARD, J. G. Semen collection and analysis in nondomestic carnivores, In: FOWLER, M. E. Zoo and Wild Animal Medicine. V. 3, p. 390- 399, 1993. Disponível em:

JIMÉNEZ, E., PÉREZ-MARÍN, C. C., MILLÁN, Y., \& AGÜERA, E. Influence of anaesthetic drugs on the epididymal sperm quality in domestic cats. Animal reproduction science, v. 123, n. 3-4, p. 265-269, 2011. Disponível em: https://doi.org/10.1016/j.anireprosci.2011.01.006

JOHNSON, A. K. Assisted Reproduction in the Male Cat. Veterinary Clinics of North America: Small Animal Practice, 2018.

KAMIBAYASHI, T.; MAZE, M. Clinical Uses of $\alpha-2$ adrenergic agonists. Anesthesiology, Philadelphia, v.93, n.5, p.1345-1349, 2000. Disponível em: https://doi.org/10.2344/0003-3006-62.1.31

KHEIRKHAH, M. S; SISAKHT, M. M.; MOHAMMADSADEGH, M.; MOSLEMI.SPERM H. R. Evaluation of Jungle Cat (Felis chaus) obtained by urethral catheterization (CT) after medetomidine administration. Theriogenology v. 91, p. 1720, 2017. Disponível em: https://doi.org/10.1016/j.theriogenology.2016.12.034

LEME, D.P.; VISACRE E.; CASTRO, V.B.; LOPES, M.D Testicular cytology by fine needle aspiration in domestic cats. Theriogenology, $v$ 106, p 46-52, 2018. Disponível em: https://doi.org/10.1016/j.theriogenology.2017.10.012

LUEDERS, I., LUTHER, I., SCHEEPERS, G., \& VAN DER HORST, G. Improved semen collection method for wild felids: Urethral catheterization yields high sperm quality in African lions (Panthera leo). Theriogenology, v. 78, n. 3, p. 696-701, 2012. Disponível em: https://doi.org/10.1016/j.theriogenology.2012.02.026

LUVONI, G.C.; KALCHSCHMIDT, E.; LEONI, S.; RUGGIERO, C. Conservation of feline semen. Part I: cooling and freezing protocols. Journal of Feline Medicine and Surgery, p. 1-6, 2003. 
MARTINS, M. I. M.; JUSTINO, R. C. Criopreservação espermática em felinos: estado da arte. Revista Brasileira de Reprodução Animal, Belo Horizonte, v. 39, n. 1, p. 136-140, 2015.2 Disponível em: http://cbra.org.br/pages/publicacoes/rbra/v39n1/pag136-140.

MORAES, M. F. D.; SILVE, M. X.; ALBUQUERQUE, A. C. A.; SILVA, A. C.; TEBALDI, J. H.; LUX-HOPPE, E. G. Helmintos de pequenos felinos selvagens no Parque Nacional do Iguaçu, Brasil. ARS Veterinária, Jaboticabal, v. 31, n. 2, p. 57, 2015. Disponível em: http://dx.doi.org/10.15361/2175-0106.2015v31n2p57

MORATO, R. G.; GUIMARÃES, M. A. B. V.; NUNES, A. L. V.; TEIXEIRA, R. H.; CARCIOFI, A.; BARNABE, V. H.; BARNABE, R. C. Colheita e avaliação espermática em onça pintada. Brazilian Journal of Veterinary Research and Animal Science, v. 35, n.4, p. 198-201, 1998. Disponível em: http://dx.doi.org/10.1590/S1413-95961998000400007

MORATO, R. G.; GUIMARÃES, M. A. B. V.; FERREIRA, F.; VERRESCHI, I. T. N.; BARNABE, R. C. Reproductive characteristics of captive male jaguar. Brazilian Journal of Veterinary Research and Animal Science, v. 36, n.5, 1999. Disponível em: http://dx.doi.org/10.1590/S1413-95961999000500008.

MORATO, R. G.; CONFORTI, V. A.; AZEVEDO, F. C. C.; JACOMO, A. T. A.; SILVEIRA, L.; SANA, D.; NUNES, A. L. V.; GUIMARÃES, M. A. B. V.; BARNABE, R. C. Comparative endocrine-ejaculate characteristics of captive versus free livingjaguars (Panthera onca). Reproduction, v. 122, n.5, p. 745-751, 2001. Disponível em: http://www.locus.ufv.br/bitstream/handle/123456789/8223.

MORATO, R. G.; VERRESCHI, I. T. N.; GUIMARAES, M. A. B. V.; CASSARO K.; PESSUTTI, C.; BARNABE, R. C. Seasonal variation in the endocrine-testicular function of captive jaguars (Panthera onca). Theriogenology, v. 61, n.7-8, p. 1273- 1281, 2004. Disponível em: https://doi.org/10.1016/j.theriogenology.2003.07.011.

PAIVA, A. L. C.; NUNES, T. L.; OLIVEIRA, M. G. C.; MORAIS, A. M. L.; SANTOS E. A. A.; SILVA A. R.; OLIVEIRA, M. F.; PAULA, V. V. Effects of atipamezole and medetomidine administration on seminal variables and functions of erection and ejaculation of the collared peccary (Tayassu tajacu) after electroejaculation. Veterinary Research, v. 8, n. 10, p. 170, 2014. doi: 10.1186/s12917-014-0170-2.

PIRES, M. V. M; SILVEIRA, A. M. M; VIEIRA, D. K.; CASTRO, J. L.C; LIBONATI, J. FERREIRA, A. M. Coleta de semen em felinos domésticos (felis catus) com o protocolo de propofol e anestesia epidural. Revista Eletrônica Novo Enfoque, v. $09, \quad$ n. 09 , p. $10-13$, 2010. Disponível em: http://www.castelobranco.br/sistema/novoenfoque/webroot/files/09/resumo/03.pd f

PISU M. C.; PONZIO, P.; ROVELLA, C. Usefulness of an injectable anaesthetic protocol for semen collection through urethral catheterisation in domestic cats Jounal of feline medicine and surgery. Journal of feline medicine and surgery, v. 19, $\mathrm{n}$. 10, p. 1087-1090, 2017. Disponível em: https://doi.org/10.1177/1098612X16679589 
POPE, C. E. Embryo technology in convservation efforts for endangered felids. Theriogenology, v 53, 163-174, 2000. Disp.onível em: https://doi.org/10.1016/S0093-691X(99)00249-6

SIEMIENIUCH, M. J.; WOCLAWEK-POTOCKA, I. Assessment of selected quality

parameters of epididymal cat (Felis catus s. domestica, L. 1758) sperm using flow cytometry method and computer assisted sperm analyser. Reproduction in domestic animals, v. 43, n. 5, p. 633-637, 2008. Disponivel em: https://doi.org/10.1111/j.1439-0531.2007.00958.x

SILVA, A. R.; MATOS, M. R. F.; CARDOSO, R. C. S; SILVA, T. F. P.; UCHOA, D.; FILGUEIRA, K. D.; et al.,; Avaliação Andrológica de uma Onça Pintada (Panthera onça) Oriunda da Região Norte do Brasil. Revista Brasileira de Reprodução Animal, v. 27, n.2, p. 217-218, 2003.

SILVA, C.A.O.; PERRI, S.H.V.; KOIVISTO, M.B.; SILVA, A.M.; CARVALHO, R.G.; MONTEIRO, C.M.R. Aspectos histológicos e morfométricos dos testículos de gato doméstico (Felis catus). Pesquisa veterinária brasileira, v. 29. n.4. p.312316, 2009. Disponível em: http://www.scielo.br/pdf/pvb/v29n4/a06v29n4.

SILVA, T. F. P.; ACKERMANN, C. L.; SILVA, L. D. M. Desafios para o desenvolvimento da tecnologia da criopreservação de sêmen felino. Ciência Animal, v. 22, n. 1, p. 143-160, 2012.

TAJIMA, H.; YOSHIZAWA, M.; SASAKI S.; YAMAMOTO, F.; NARUSHIMA E.; OGAWA, Y.; et al.,; A trial of semen collection by transrectal electroejaculation method from Amur leopard cat (Prionailurus bengalensis euptilurus). The Journal of Veterinary Medical Science, v. 78, n. 6, p. 1067-1073. doi: 10.1292/jvms.15-0439

TAJIMA, H.; YOSHIZAWA, M.; SASAKI, S.; YAMAMOTO, F.; NARUSHIMA, E.; TSUTSUI, T.; et al.,; Intrauterine insemination with fresh semen in Amur leopard cat (Pionailurus bengalensis eutilura) during non-breeding season. The Journal of Veterinary Medical Science, v. 79, n. 1, p. 92-99, 2017. Disponível em: 10.1292/jvms.15-0683

TRIGO, T. C., SCHNEIDER, A., DE OLIVEIRA, T. G., LEHUGEUR, L. M., SILVEIRA, L., FREITAS, T. R., \& EIZIRIK, E. Molecular data reveal complex hybridization and a cryptic species of Neotropical wild cat. Current Biology, v.23 n. 24 p. 2528-2533. 2013. https://doi.org/10.1016/j.cub.2013.10.046

TEBET, J. M., MARTINS, M. I. M., CHIRINEA, V. H., SOUZA, F. F. D., CAMPAGNOL, D., \& LOPES, M. D. Cryopreservation effects on domestic cat epididymal versus electroejaculated spermatozoa. Theriogenology, v. 66, n. 6-7, p. 1629-1632, 2006. https://doi.org/10.1016/j.theriogenology.2006.02.013 
TOYONAGA, M., SATO, Y., MORITA, M., WATANABE, M., OBA, H., MIZUTANI, T., ... \& TSUTSUI, T. The qualities of cryopreserved epididymal sperm collected from feline epididymides stored at low temperature. Journal of Veterinary Medical Science, v. $72, \quad$ n. $6, \quad$ p. 777-780, 2010. Disponivel em: https://doi.org/10.1292/jvms.09-0260

VALIENTE, C.; SOTA, P.; ARAUZ, S. Ejaculation training, seminal alkaline phosphatase and semen preservation through cooling in a milk-based extender in domestic cats Jounal of feline medicine and surgery. Journal of feline medicine and surgery, v. $16, \quad n .4, \quad$ p. $312-316,2014$. Disponivel em: https://doi.org/10.1177/1098612X13508192

ZAMBELLI, D.; PRATI, F.; MERLO, B.; CUNTO, M. Collection of semen by urethral catheterization after pharmacologically induced spermatozoa releasing in the domestic cat. in: Proceedings of the $5^{\text {th }}$ Biannual Congress. European Veterinary Society for Small Animal Reproduction, 2006.

ZAMBELLI, D.; PRATI, F.; MERLO, B. Effects of ketamine or medetomidine administration on quality of electroejaculated sperm and on sperm flow in the domestic cat. Theriogenology. v 68, n 5, p 796-803, 2007.

ZAMBELLI D, PRATI F, CUNTO M, IACONO E, MERLO B. Quality and in vitro fertilizing ability of cryopreserved cat spermatozoa obtained by urethral catheterization after medetomidine administration. Theriogenology, 69:485-90, 2008. 